\title{
Regression Model Method for Analyze the Association Rules using Major Parameters
}

\author{
G. G. Shah, H. N. Patel
}

\begin{abstract}
Using the data mining user can extract the information. Frequent itemsets is one of the popular task in data mining. Association Rule Analysis is the task of discovering association rules that occur frequently in a given large data set.The task is to find certain relationships among a set of itemsets in the database. There are two fundamental parameter(measurement) is Support and Confidence.Traditional association rule mining techniques employ predefined support and confidence values. But, it's observed that specifying minimum support value of the minded rules in advance often leads to either too many or too few rules, which negatively impacts the performance of the overall system.This paper proposes a non-linear regression model using support, confidence and association rules. To predict the number of rules under the given explanatory variables say parameters. Use the $R$ language for the Rules generations and also uses significance test to verify regression coefficients. Using the coefficient test and F-test verify the model.
\end{abstract}

Keywords: Association Rules, Regression, Regression Coefficients, Multiple Correlation, F-test

\section{INTRODUCTION}

Data Mining is a process of extraction of useful information from the large database and pattern from the huge data [1]. Data Mining is a logical process for to search large amount of data in order to find useful data. The aim of this technique is to find patterns that were previously unknown. There are various techniques like Classification, Clustering, Regression, Artificial Intelligence, Neural Networks, Association Rules, Decision Tree, Generic Algorithm, Nearest Neighbor method etc., are used for knowledge discovery from database.

Association Rule is one of the most popular techniques and an important research issue in the area of Data Mining [2].Association Rule is usually to find frequent items from the large data.It is helpful for findings helps businesses to make certain decisions like shopping behavior analysis in the super mall, catalogue design, cross marketing, fraud detections, finance, telecommunication etc. However the number of Association Rules for a given dataset is generally very large.

To improve the efficiency of the Rules various researchers have developed the frameworks and algorithms. These include Agrawal et al.[3] have developed AIS, SETM, Apriori, Apri-oriTid to discover significant association rules between items in large data.

Revised Manuscript Received on May 20, 2020.

G. G. Shah, Faculty of Business Administration, Dharmsinh Desai University, Nadiad, India. E-mail: gopalgshah16@yahoo.com

Dr. H. N. Patel, Department of Computer Science, Dr. Babasaheb Ambedker Open University, Ahmedabad, India. E-mail: himanshu.patel@baou.edu.in
Number of algorithms are developed and compare with above define algorithms and among them areDHP(Park, Chen et al., 1995), CHARM (Zaki and Hsiao, 1999), FP Growth ( Han, Pei et al.,, 2000), RARM (Das, Ng et al., 2001), Closet+ (Wang, Han et al., 2003) etc.

In the last two decades, little work has been done on how to choose both appropriate thresholds of support and confidence for the mining algorithms before a real mining process. It is observed the lack of approaches or techniques or mechanism for thresholds selection of support and confidence and it often leads to either too many or too few rules after completing a mining process. Also it can define or lead to either excessive computation time or poor results. Therefore, current association rule mining technique or approaches are facing following challenges

a). In the Particular Mining algorithm selection of the thresholds of Support and Confidence. b). Extension of the particular approach using the Matrix Application.c). Reduce the time needed for large dataset. In this research paper proposed approach consists of

(i). Generated Regression Model consists of Support, Confidence and Association Rules as an explanatory variable and Response variable.(ii). The Model is applicable for a particular domain.

Therefore, the proposed approach can be applied to different domains with different types of datasets to select thresholds of support and confidence in an association rule mining algorithm. Also, it demonstrates a case study to show the effective performance of the proposed approach on a realworld dataset in support and confidence selection.

The rest of this paper includes as: Section 2 introduces the Regression Model Approach, Section 3 contains the Matrix application for the Regression, Section 4 contains the Regression Statistics, and Section 5 concludes with Conclusions.

\section{REGRESSION MODEL APPROACH}

In this section, the proposed approach is based on Regression Approach. A regression model is generated in a general level by number of association rules, and support coefficient and confidence coefficient.Naturally in principle the number of association rules in a dataset depends upon the support coefficient and confidence coefficient.

The formula of support and confidence for the form $A \Rightarrow B$ can be formally define as follow.

$$
\begin{gathered}
\text { Support }(A \Rightarrow B)=P(A \cup B) \\
\text { Confidence }(A \Rightarrow B)=P(A \backslash B)
\end{gathered}
$$

Note:

i.The value of the support and confidence are lies in between 0 and 1. 


\section{Regression Model Method for Analyze the Association Rules using Major Parameters}

ii. Support describe the significance of the rule and Confidence describe the strengths of the rule.

The Regression Model to be define using Association Rules, Support and Confidence are as follow

$$
y=\beta_{0}+\beta_{1} x_{1}+\beta_{2} x_{2}+\beta_{3} \frac{1}{x_{1}}+\beta_{4} \frac{1}{x_{2}}
$$

Where $\mathrm{y}$ is the number of association rules, $x_{1}$ and $\frac{1}{x_{1}}$ represents the support variable and its reciprocal and similarly $x_{2}$ and $\frac{1}{x_{2}}$ represents the confidence variable and its reciprocal respectively. $\beta_{0}, \beta_{1}, \beta_{2}, \beta_{3}$ and $\beta_{4}$ are coefficients of the regression model. The coefficient $\beta_{1}$ and $\beta_{3}$ measures the partial effect of $x_{1}$ and $x_{3}$ ony. Similarly, The coefficient $\beta_{2}$ and $\beta_{4}$ measures the partial effect of $x_{2}$ and $x_{4}$ ony,the coefficient $\beta_{0}$ is rarely explained and evaluated in a regression model.It is known as intercept and it includes all other factors except support and confidence. Using the equation (1) we can see that the number of association rules depends on values of support and confidence, also $\beta_{1}$ and $\beta_{2}$ in a particular domain.After taking the simple variable transformation the equation (1) can be define as $y=\beta_{0}+\beta_{1} X_{1}+\beta_{2} X_{2}+\beta_{3} X_{3}+\beta_{4} X_{4}$

\section{MATRIX APPLICATION FOR THE REGRESSION}

Given the actual data values, we may write the model for $n$ number of association rules of the above type, where $i^{\text {th }}$ association is

$$
\begin{aligned}
& Y_{i}=\sum_{j=1}^{k} \beta_{j} X_{j} j=1,2 \ldots, \text { kandi }=1,2, \ldots, n \\
& \text { Which can be written in matrix notation as }
\end{aligned}
$$

$$
\underline{Y}=\underline{\beta} X+\epsilon
$$

Where

$$
\begin{aligned}
& Y=\left(y_{1}, y_{2}, \ldots, y_{n}\right)^{T}, \epsilon=\left(\epsilon_{1}, \epsilon_{2}, \ldots, \epsilon_{n}\right)^{T}, \beta= \\
& \left(\beta_{0}, \beta_{1}, \ldots, \beta_{n}\right)^{T} \text { And } \\
& A=\left[\begin{array}{ccccc}
X_{11} & X_{12} & \ldots & \ldots & X_{1 n} \\
X_{21} & X_{22} & \ldots & \ldots & X_{2 n} \\
\ldots & \ldots & \ldots & \ldots & \ldots \\
\ldots & \ldots & \ldots & \ldots & \ldots \\
\ldots & \ldots & \ldots & \ldots & \ldots \\
X_{n 1} X_{n 2} & \ldots & \ldots & X_{n n}
\end{array}\right]
\end{aligned}
$$

We can assume that $E(\epsilon)=0$ and $\mathrm{V}(\epsilon)=$ $\sigma^{2}$ Isince if this were not so, we could simply absorb the nonzero expectation for the error into the mean $\mu$ to get a zero expectation.

\section{A. Estimating $\boldsymbol{\beta}$}

We have to obtain estimates of $\beta_{1}, \beta_{2}, \ldots \ldots, \beta_{k}$. The regression $Y=X \beta+\epsilon$, partitions, the response into a systematic component $X \beta$ and a random component $\epsilon$. The problem is to find $\beta$ so that $X \beta$ is as close to the response variable $\mathrm{X}$ is as close to the response variable $\mathrm{Y}$ as possible. The cure method for estimate $\hat{\beta}$, to be define in the following geometrical figure.

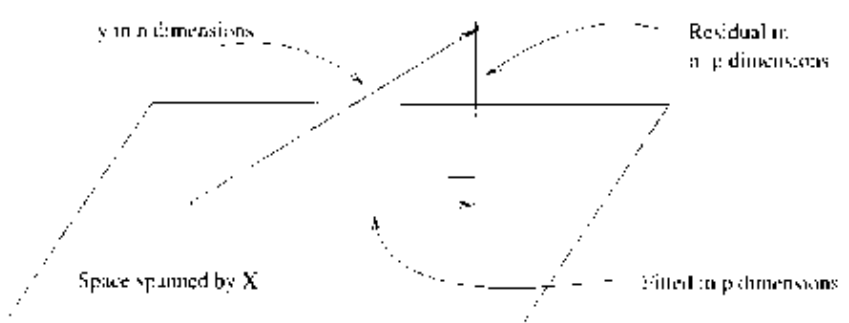

Figure 1 Regression Coefficient

In the model space, $\hat{\beta}$ is the best method to estimate $\beta$. The response variable $\mathrm{Y}$ to be predicted by the model $\hat{Y}=X \hat{\beta}$. The difference between the actual response variable and the predicted response is denoted by $\hat{\epsilon}$ and it is known as residuals.

The conceptual purpose of the generalized model is to represent, an accurately as possible. The response variable y is n-dimensional, in terms of very simple way the model, which is k-dimensional.

So, if model is much successful, the structure in the data should be define by $\mathrm{k}$ - dimensional and from the above figure it is clear that the random variation in the residuals are lie in (n-k) dimensional space.

\section{B. LEAST SquARES ESTIMATION}

We have to obtain estimates of $\beta_{0}, \beta_{1}, \beta_{2}, \beta_{3}, \beta_{4}$ from a nongeometrical point of view. We might define the best estimate $\beta$ as the one which minimize the sum of the squared errors. The least squares estimate of $\beta$, called $\hat{\beta}$.

$$
\begin{gathered}
\underline{Y}=X \hat{\beta}+\underline{\epsilon}(3.2 .1) \\
\underline{\epsilon}=\underline{Y}-X \hat{\beta}
\end{gathered}
$$

Considering sum of squares of errors, that is $\sum \epsilon_{i}{ }^{2}=\epsilon^{T} \epsilon=(Y-X \hat{\beta})^{T}(Y-X \hat{\beta})=Y^{T} Y-2 \hat{\beta}^{T} X^{T} Y+$ $\hat{\beta}^{T}+\hat{\beta}^{T}\left(X^{T} X\right) \hat{\beta}$

Differentiating with respect to $\hat{\beta}$ and setting to zero, we find that $\hat{\beta}$ satisfies:

We have,

$$
\frac{\partial\left(\epsilon^{T} \epsilon\right)}{\partial \widehat{\beta}}=-2 X^{T} Y+2\left(X^{T} X\right) \hat{\beta}=0
$$

$$
X^{T} Y=\left(X^{T} X\right) \hat{\beta}(3.2 .3)
$$

These are called the normal equation. We have to solve them. Let $X^{T} X$ be positive definite matrix, that is $\left|X^{T} X\right| \neq$ 0. In this case pre-multiplying both side of $X^{T}$ we get

$$
\widehat{\beta}=\left(X^{T} X\right)^{-1} X^{T} Y
$$

And if $\left|X^{T} X\right|=0$ then the solution of (3.2.2) does not exists, but by taking generalized inverse $\left(X^{T} X\right)^{-1}$ of $\left(X^{T} X\right)$ then the generalized solution is

$$
\widehat{\beta}=\left(X^{T} X\right)^{-1} X^{T} Y
$$

\section{REGRESSION STATISTICS}

In the given regression model generation Response Variable is defined by $\hat{y}$. The Explanatory Variable is define by $X_{i}, i=1,2,3,4$. Using the dataset, the obtain Rules are denoted by Response Variable and Support and Confidence are define by Explanatory Variable. Support and Confidence are in the inverse relation with Association Rules, so generate the non-linear regression model. In the above section define the formula for the Regression Coefficient. By

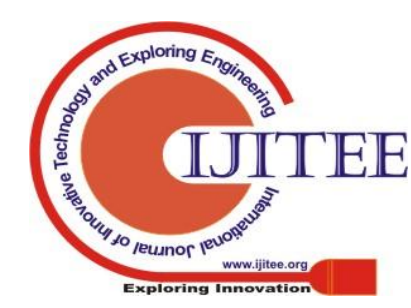


using matrix calculation obtain Regression Coefficients.

Table 1. Association Rules

\begin{tabular}{|c|c|c|c|c|}
\hline Rules & Supp & Conf & $\boldsymbol{x}_{\mathbf{1}}$ & $\boldsymbol{x}_{\mathbf{2}}$ \\
\hline 27 & 0.1 & 0.5 & 10 & 2.000 \\
\hline 24 & 0.1 & 0.55 & 10 & 1.818 \\
\hline 20 & 0.1 & 0.6 & 10 & 1.667 \\
\hline 18 & 0.1 & 0.65 & 10 & 1.538 \\
\hline 17 & 0.1 & 0.7 & 10 & 1.429 \\
\hline 22 & 0.15 & 0.5 & 6.667 & 2.000 \\
\hline 19 & 0.15 & 0.55 & 6.667 & 1.818 \\
\hline 15 & 0.15 & 0.6 & 6.667 & 1.667 \\
\hline 14 & 0.15 & 0.65 & 6.667 & 1.538 \\
\hline 13 & 0.15 & 0.7 & 6.667 & 1.429 \\
\hline 8 & 0.2 & 0.5 & 5 & 2.000 \\
\hline 6 & 0.2 & 0.55 & 5 & 1.818 \\
\hline 5 & 0.2 & 0.6 & 5 & 1.667 \\
\hline 5 & 0.2 & 0.65 & 5 & 1.538 \\
\hline 5 & 0.2 & 0.7 & 5 & 1.429 \\
\hline
\end{tabular}

Using the above Table 1, to obtain the Regression Coefficients. Convert the specific columns into Response and Explanatory Variable. Rules are define as the Response and Supp, Conf, $x_{1}$ and $x_{2}$ are define as Explanatory Variables. They are convert into Matrix form and define in the following steps:

Step 1. Define Rules as Response Variable by y (shown below)

Step 2. Define Support, Confidence, $x_{1}, x_{2}$ as Explanatory Variable by $\mathrm{X}$. (shown below)

$$
y=\left[\begin{array}{c}
27 \\
24 \\
20 \\
18 \\
17 \\
22 \\
19 \\
15 \\
14 \\
13 \\
8 \\
6 \\
5 \\
5 \\
5
\end{array}\right] X=\left[\begin{array}{cccc}
0.1 & 0.5 & 10 & 2.000 \\
0.1 & 0.55 & 10 & 1.818 \\
0.1 & 0.6 & 10 & 1.667 \\
0.1 & 0.65 & 10 & 1.538 \\
0.1 & 0.7 & 10 & 1.429 \\
0.15 & 0.5 & 6.667 & 2.000 \\
0.15 & 0.55 & 6.667 & 1.818 \\
0.15 & 0.6 & 6.667 & 1.667 \\
0.15 & 0.65 & 6.667 & 1.538 \\
0.15 & 0.7 & 6.667 & 1.429 \\
0.2 & 0.5 & 5 & 2.000 \\
0.2 & 0.55 & 5 & 1.818 \\
0.2 & 0.6 & 5 & 1.667 \\
0.2 & 0.65 & 5 & 1.538 \\
0.2 & 0.7 & 5 & 1.429
\end{array}\right]
$$

Step 3. Obtain Transpose of the given matrix X.

Step 4. Find $\left(X^{\prime} X\right)$

Step 5. Find Inverse of $\left(X^{\prime} X\right)$ say $\left(X^{\prime} X\right)^{-1}$

Step 6. Find $X^{\prime} Y$

Step 7. Find $\left(X^{\prime} X\right)^{-1} X^{\prime} Y$ and denoted as $\hat{\beta}$.

$$
\hat{\beta}=\left[\begin{array}{c}
-340.76 \\
58.981 \\
-3.735 \\
33.859
\end{array}\right]
$$

\section{Interpretation:}

In the above matrix the Coefficient define as with the value $340.76,58.981,-3.735$ and 33.859 respectively. The coefficient $x_{1}$ is define for Support and $x_{3}$ is the inverse of Support. The sign of $x_{1}$ and $x_{3}$ are negative, that means if the independent variable increases, then the value of the dependent variable tends to decreases.

Similarly $x_{2}$ is define for Confidence and $x_{4}$ is the inverse of the Confidence. Here, $x_{2}$ and $x_{4}$ are both positive. The value of $x_{2}$ are increase then the value of the independent variable increase.

It means in the above the data, the Response variable say number of rules and Explanatory variable say Support are inverse related with each other. If the Support increase the number of rules are decrease but negative sign indicatesthat the both variable are opposite direction. Here, the obtain Regression Model is

$$
y=-340.76 X_{1}+58.981 X_{2}-3.735 X_{3}+33.859 X_{4}
$$

\section{A. REGRESSION MODEL TEST}

Before predicting the number of association rules, the define regression model satisfies the following standard evaluation or not.

\section{i. $\quad$ Multiple Correlation Coefficient evaluation}

The detail calculation of $R$ is shown as follows.

$$
R=\sqrt{1-\frac{\sum\left(y_{i}-\hat{y}_{i}\right)^{2}}{\sum\left(y_{i}-\bar{y}_{i}\right)^{2}}}=0.9848
$$

It means that there is a strong relationship between the number of rules and support and confidence in the given dataset.

\section{B. REgRESSION MODEL TEST}

Now we have to test for the significance of the regression model. If the model is satisfied by test, we need to carry out the second test. In other cases, the data sample is considered by increasing the number of observations. The second test is to test individual regression coefficients $\beta_{1}, \beta_{2}, \beta_{3}$ and $\beta_{4}$.here, $\beta_{0}$ define as the constant so it is not need to be evaluated. The null hypothesis for the regression model is

$$
\begin{gathered}
H_{0}: \beta_{1}=\beta_{2}=\beta_{3}=\beta_{4}=0 \text { aganist } \\
H_{1}: \beta_{1} \neq \beta_{2} \neq \beta_{3} \neq \beta_{4} \neq 0 \\
R^{2}=0.9699, \text { and } \\
F=\frac{R^{2} / k}{\left(1-R^{2}\right) /(n-k-1)}=\frac{0.9699 / 4}{(1-0.9699) /(15-4-1)}=80.55
\end{gathered}
$$

The critical value for this test, corresponding to a significance level of $5 \%$ is

$F_{\alpha(k-1, n-k-1)}=F_{0.05,3,10}=3.71$.Therefore,

$F_{\text {cal }}>$

$F_{\alpha(2, n-3)}$. Null Hypothesis $H_{0}$ is rejected and it is concluded that at least one coefficient among $\beta_{1}, \beta_{2}, \beta_{3}$ and $\beta_{4}$ is significant. In other words, there exists the relationship between the number of association rules and either support factor, confidence factor or both of support and confidence. 


\section{Regression Model Method for Analyze the Association Rules using Major Parameters}

\section{Regression Coefficient Test}

The aim of the Regression Coefficient Test is to observe which factors (support, confidence) exist in the regression model.

(i) The null hypothesis to test $\beta_{1}$ is

$$
\begin{gathered}
H_{0}: \beta_{1}=0 \text { aganist } H_{1}: \beta_{1} \neq 0 \\
t=\frac{\hat{\beta}_{1}-\beta_{1}}{S E\left(\hat{\beta}_{1}\right)}
\end{gathered}
$$

Where standard error of the regression coefficients are obtained from the variance-covariance matrix say $\left(X^{T} X\right)^{-1}$ and $\hat{\sigma}$.Similarly $\hat{\sigma}$ is known as standard error of the model and it is obtained as

$$
\begin{gathered}
\hat{\sigma}=\sqrt{\frac{\sum \epsilon_{i}{ }^{2}}{n-k-1}} \\
t=\frac{\hat{\beta}_{1}-\beta_{1}}{S E\left(\hat{\beta}_{1}\right)}=\frac{\hat{\beta}_{1}-\beta_{1}}{\sqrt{d_{11}} \hat{\sigma}}=\frac{-340.0744-0}{\sqrt{1120.865} \times 1.511307} \\
=-6.7211
\end{gathered}
$$

The critical values of $t_{(0.05,15-4-1)}=t_{(0.05,10)}$ with a significance of 0.05 are 2.228 respectively. Since $t=$ $6.7211>t_{(0.05,10)}$, the null hypothesis $H_{0}$ is rejected and it states that the number of association rule is effected by support.

$$
\begin{aligned}
& \text { ii) The null hypothesis to test } \beta_{2} \text { is } \\
& H_{0}: \beta_{2}=0 \text { aganist } H_{1}: \beta_{2} \neq 0 \\
& t=\frac{\hat{\beta}_{2}-\beta_{2}}{S E\left(\hat{\beta}_{2}\right)}=\frac{\hat{\beta}_{2}-\beta_{2}}{\sqrt{d_{22}} \hat{\sigma}}=\frac{62.00854-0}{\sqrt{1342.353} \times 1.511307} \\
& =1.1199
\end{aligned}
$$

The critical values of $t_{(0.05,15-4-1)}=t_{(0.05,10)}$ with a significance of 0.05 are 2.228 respectively. Since $t=$ $1.1199<t_{(0.05,10)}$, the null hypothesis $H_{0}$ is accepted and it states that the number of association rule is not effected by confidence.

\section{CONCLUSIONS}

Using the generated non-linear Regression model it is clear that Support and Confidence are the fundamental parameter for the Association Rules. In the proposed a new approach the association rule mining process through the prediction of the potential number of association rules on dataset. In our approach is designed and evaluated by Multiple Correlation, Regression Model and Regression Coefficient tests in terms of significance of Support and Confidence in the model.To consider which factor exists in the define Regression Model, it is to be define by the coefficient test. In the future study, our approach to datasets in other general areas like Transportation, Telecommunication, Mutual Funds, and Finance and so on.

\section{REFERENCES}

1. B. Ramageri, "DATA MINING TECHNIQUE AND APPLICATIONS," Indian Journal of Computer Science and Engineering, 2010.

2. D. T. Le, F. Ren, and M. Zhang, "A Regression-Based Approach for Improving the Association Rule Mining through Predicting the Number of Rules on General Datasets," Lecture Notes in Computer Science PRICAI 2012: Trends in Artificial Intelligence, pp. 229-240, 2012.

3. Agrawal, R., Imieliński, T. and Swami, A. (1993). Mining association rules between sets of items in large databases. ACM SIGMOD Record, 22(2), pp.207-216

\section{AUTHORS PROFILE}

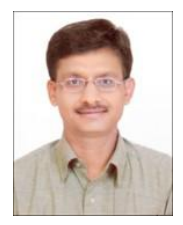

G. G Shah, Assistant Professor at Dharmsinh Desai University. Having Experience of 21 at U.G Level.. Published 4 research articles at National and International journals. The main interest field is Data Science

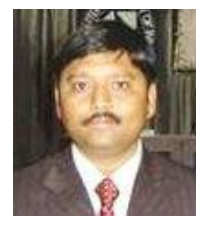

Dr.H.N Patel, Assistant Professor at Dr. BabasahebAmbedkar Open University. Having experience of 15 years. 4 Paper published in International Journal and 5 Paper published in National Journal with ISSN/ISBN no. and Total 11 Paper presented in Conference/Seminar/ Workshop. He is having Lifetime membership of CSI and was member of ACM. 